Ann. Zootech., 1985, 34 (3), 291-312

\title{
Comparaison entre espèces (mouton, cheval, bovin) de la digestibilité et des quantités ingérées des fourrages verts
}

\author{
M. CHENOST, W. MARTIN-ROSSET
}

avec la collaboration technique de J.M. BOISSAU, Madeleine DUDILIEU, Marie JAILLER, L. L'HOTELIER, H. DUBROEUCQ, R. JAILLER, G. MOINS

Laboratoire des Aliments, Unité sur l'Elevage du Cheval I.N.R.A., C.R.Z.V. de Theix, 63122 Ceyrat

\section{Résumé}

Les données bibliographiques relatives aux études comparatives de la digestibilité et des quantités volontairement ingérées entre espèces animales portent surtout sur des fourrages conservés et ont été obtenues essentiellement sur ruminants (tabl. 1).

L'objectif de notre étude était 1) de vérifier sur un ensemble de fourrages verts dont la «digestibilité mouton» variait dans des limites très larges (de 60,6 à 76,6 p. 100), tableau 2, si les différences entre espèces animales présentaient les mêmes tendances que sur fourrages conservés; 2) d'ajouter aux ruminants (moutons, taurillons, vaches laitières) un autre herbivore non ruminant, le cheval, pour lequel il existe très peu de données.

Les fourrages verts étaient distribués à l'auge, à volonté (refus de 10 à 15 p. 100), suivant le dispositif porté au tableau 2. Les mesures de digestibilité duraient 6 jours. Lorsqu'elles étaient effectuées en continu au cours d'un cycle, une période de mesure donnée servait de période pré-expérimentale pour la suivante. Dans le cas de mesures isolées les animaux recevaient pendant la semaine précédant la semaine de mesure une herbe de caractéristique aussi voisine que possible de l'herbe expérimentale.

1. Les digestibilités de la matière organique (dMO) et de la cellulose brute (dCB) sont significativement plus élevées chez les bovins que chez les moutons (différences moyennes respectives de 5,9 et 9,0 points). elles-mêmes significativement plus élevées que chez le cheval (différences moyennes respectives de 3,8 et 8,3 points), tableau 3 . La digestibilité des matières azotées (dMAT) est la même chez les 3 catégories d'animaux (fig. 3). Des liaisons étroites permettent de prévoir la dMO bovins (nous n'avons pas pu séparer les taurillons des vaches laitières en raison du nombre trop faible de mesures pour chaque type d'animaux) et la dMO cheval à partir de la dMO mouton avec une bonne précision (Syx $=2,31$ points, $n=9$ et $\operatorname{Syx}=2,32$ points, $n=12$ respectivement), figure 1 . Il en est de même de la prévision de la dCB (fig. 2).

La variabilité de la dMO entre animaux est la plus faible chez le mouton et la plus élevée chez les bovins.

La dMO d'un fourrage dépend essentiellement de la digestibilité de ses parois, caractérisées par les fractions «cellulose brute» et NDF (méthode de VAN SOEST, 1967).

Malgré une efficacité de mastication plus faible chez les bovins que chez les moutons, la digestibilité des parois est meilleure chez les bovins. Cette différence doit donc vrai- 
semblablement s'expliquer par le temps de séjour des particules de fourrage dans le rumen plus long chez le bovin que chez le mouton, qui permet une dégradation cellulolytique et une digestion plus complète chez le bovin.

Les différences entre ruminants et chevaux peuvent être expliquées par une activité cellulolytique de la population microbienne plus faible et un temps de séjour des particules plus court chez le cheval que chez le ruminant.

2. Les quantités ingérées (QI) exprimées en $\mathrm{g} / \mathrm{kg} \mathrm{P}^{0,75}$ sont voisines chez les bovins et le cheval mais plus élevées que chez le mouton (tabl. 3). Il ne nous a pas été possible de relier les QI bovins ou cheval aux QI moutons. La variabilité des QI, identique chez les bovins et le cheval, est plus grande que chez le mouton.

3. Les liaisons entre d'une part $\mathrm{dMO}$ ou $\mathrm{QI}$ et d'autre part les caractéristiques du fourrage (MS, composition chimique, digestibilité) sont lâches (tabl. 5) mais cela résulte, sans doute, du fait que nous avons travaillé :

- sur un nombre encore restreint de fourrages, de caractéristiques très différentes;

- sur des animaux de stades physiologiques différents (animaux à l'entretien : mouton, cheval; en production : vaches laitières ou en croissance : taurillons).

4. Cette étude, encore fragmentaire, complète les résultats observés sur fourrages conservés. Elle permet également de commencer à décrire la nature des relations dMO mouton/dMO bovin et dMO cheval/dMO ruminant. Il est nécessaire de la poursuivre sur d'autres fourrages verts bien caractéristiques avec des animaux pris à des stades physiologiques ou à des niveaux de production comparables d'une espèce à l'autre.

Mots clés : Comparaison, interespèces, digestibilité, herbe.

\section{Introduction}

La digestibilité des aliments ou des rations est généralement mesurée sur des moutons pour des raisons pratiques (quantités de fourrages à distribuer, facilité de collecte des fèces, ...) et économiques (coût des installations et de l'alimentation). Les résultats de digestibilité obtenus sur moutons sont très souvent appliqués aux bovins. Or de nombreuses comparaisons effectuées sur fourrages conservés (tabl. 1), (CiPOLLiNi et al., 1951, mise au point de Schneiner \& FLATT, 1965) ont montré que l'aptitude digestive des bovins était généralement supérieure à celle des moutons. En revanche les études comparatives sur fourrages verts sont rares. Les fourrages conservés et l'herbe peuvent constituer l'intégralité de la ration des chevaux, herbivores comme les ruminants. Or, à part quelques mesures anciennes effectuées au début du siècle par Wolf et al. (cité par Van Der Noot \& Gilbreath, 1970), et les travaux plus récents de VAN Der NoOt, Cordts \& Hunt (1965), la digestibilité de l'herbe par le cheval reste très mal connue.

Notre étude s'inscrit dans un ensemble de travaux dont l'objectif est de comparer la digestibilité et les quantités ingérées des fourrages verts chez le mouton, les bovins et le cheval (à l'auge et au pâturage).

Nous avons voulu ici :

- vérifier si les différences de digestibilité observées entre bovins et ovins sur les fourrages conservés existent également sur les fourrages verts distribués à l'auge ;

- ajouter un autre herbivore, le cheval, dans les essais de comparaison, afin de pouvoir prévoir la digestibilité cheval à partir de la digestibilité mouton ;

- comparer également les quantités ingérées selon l'espèce animale. 


\section{Matériel et méthodes}

Au laboratoire, nous ne disposions pas d'installation spécialement conçue pour conduire des études comparatives de digestibilité simultanément sur plusieurs espèces animales. Nous avons donc été amenés à utiliser, parallèlement aux moutons de notre atelier de digestibilité, des taurillons, des vaches laitières et des chevaux appartenant à trois autres laboratoires. En raison des contraintes propres à ces derniers, les bovins et les chevaux n'ont pas toujours pu être mis simultanément à notre disposition. C'est pourquoi les fourrages expérimentaux, distribués systématiquement aux moutons, n'ont pas été tous distribués aux autres espèces à la fois.

Ainsi, sur 15 périodes expérimentales (tabl. 2), 5 seulement ont-elles été communes aux moutons, aux bovins et aux chevaux. Les autres périodes constituaient, finalement, des comparaisons soit moutons/bovins, soit moutons/chevaux. Par ailleurs, il n'a pas été possible d'achever le $3^{\mathbf{e}}$ essai avec l'ensemble moutons-chevaux-taurillons, ces derniers ayant été affectés de diarrhées et ne mangeant plus normalement.

\section{Dispositif expérimental}

Le tableau 2 résume les différents essais effectués : calendrier d'exploitation et nature des différentes prairies (caractéristiques et composition chimique de l'herbe distribuée), espèces animales utilisées.

\section{Fourrages}

L'herbe a été exploitée en affouragement en vert dans les 3 essais. Dans le premier essai, elle a été distribuée aux animaux sous forme longue. Dans les $2^{\circ}$ et $3^{\mathrm{e}}$ essais, elle a été récoltée à l'ensileuse coupe fine (brins d'environ $5 \mathrm{~cm}$ ) et donnée sous forme hachée.

Dans tous les essais l'herbe était récoltée une fois par jour en fin de matinée ou début d'après-midi pour éviter la rosée et le samedi matin pour l'affouragement du samedi et du dimanche. Elle a été distribuée à raison de 2 repas par jour (9 et $16 \mathrm{~h}$ ) pour les moutons et les vaches et de 3 repas/jour $(8,12$ et 16 h) pour les chevaux.

Les animaux qui ne recevaient aucun complément étaient alimentés à volonté (le pourcentage de refus toléré a varié entre 10 et 15 p. 100) sauf pour les deux luzernes du $3^{\circ}$ essai qui ont été distribuées à un niveau voisin de 1,5 fois l'entretien, afin d'éviter d'éventuels troubles digestifs.

\section{Animaux}

Nous avons reporté dans le tableau 2 les principales informations zootechniques relatives aux animaux utilisés au cours des 3 essais. Les chevaux (trotteurs réformés) et les moutons castrés étaient à l'entretien, les vaches laitières en milieu de lactation et les taurillons en croissance. Le nombre d'animaux par lot n'a pas été le même d'une unité expérimentale à l'autre (4 pour les vaches, 5 pour les chevaux, 6 pour les moutons et les taurillons). 


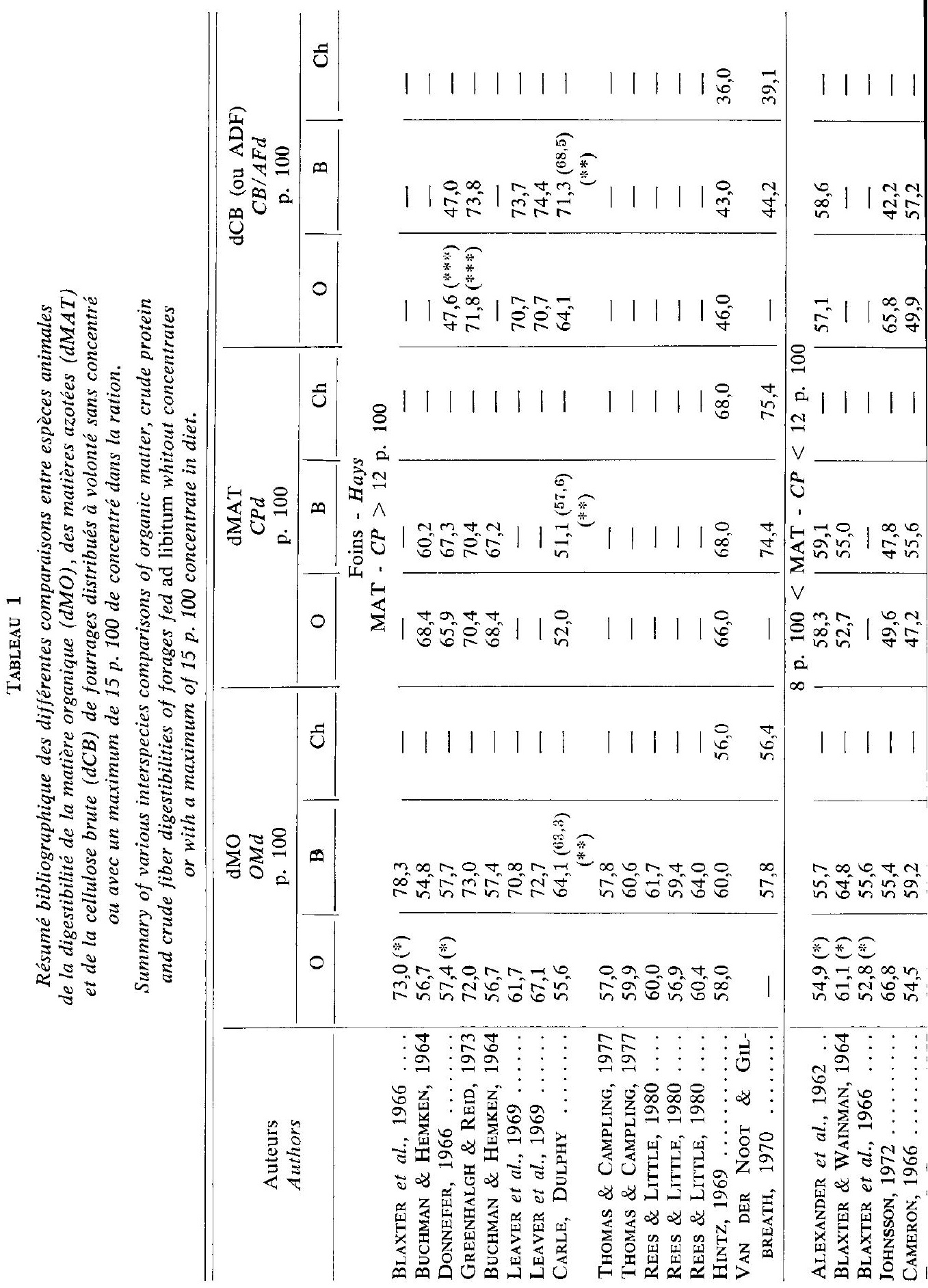




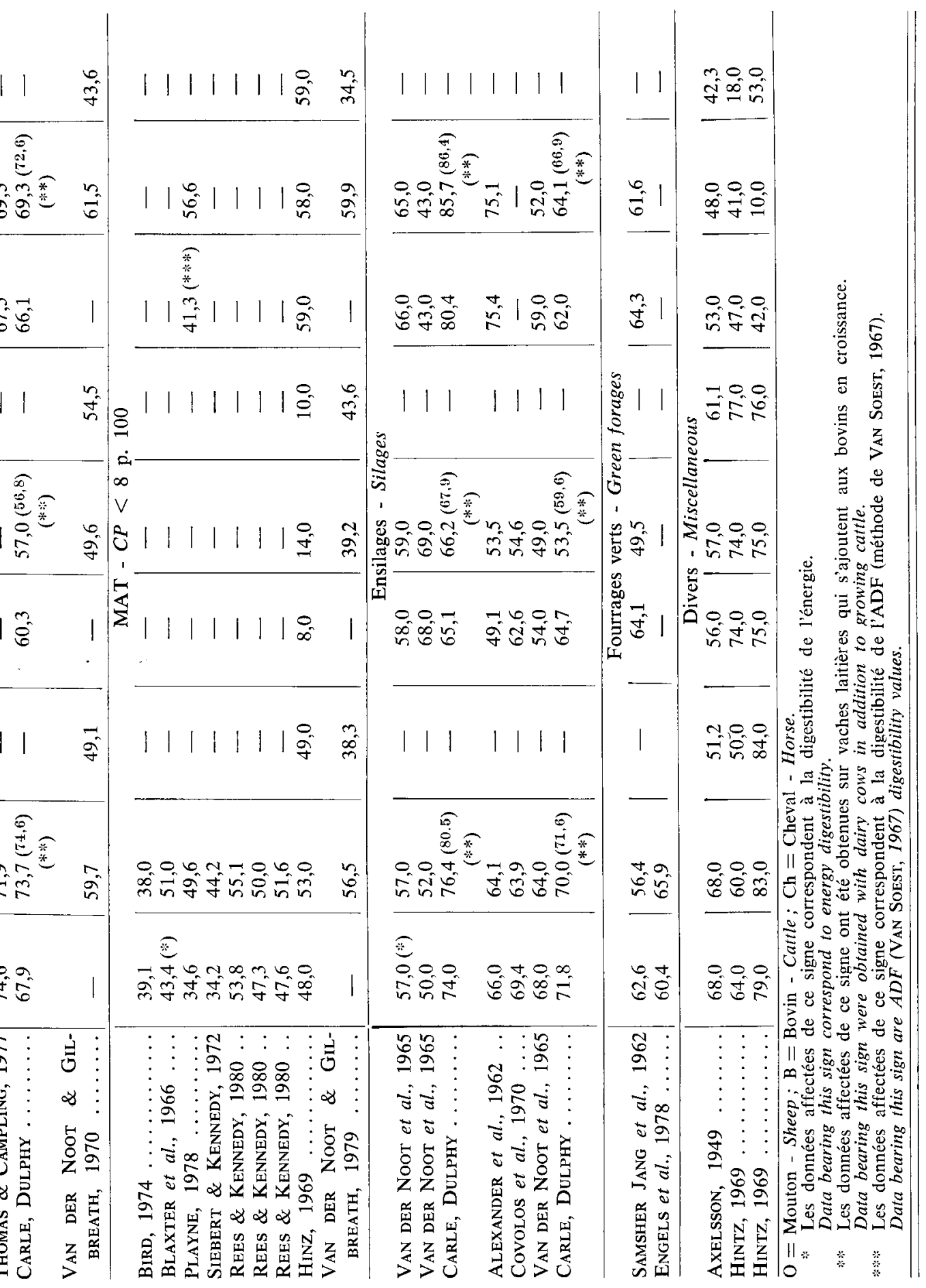




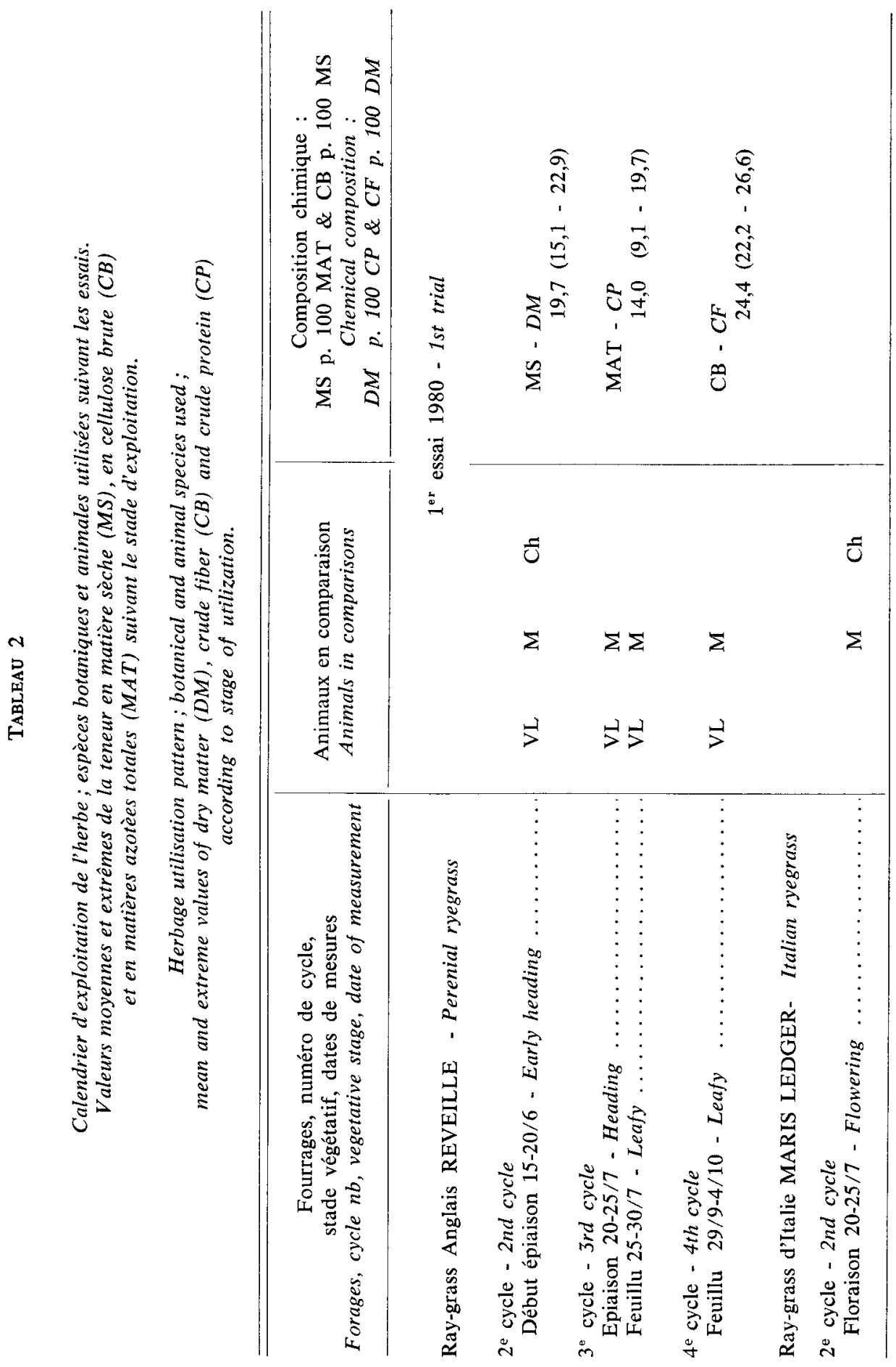




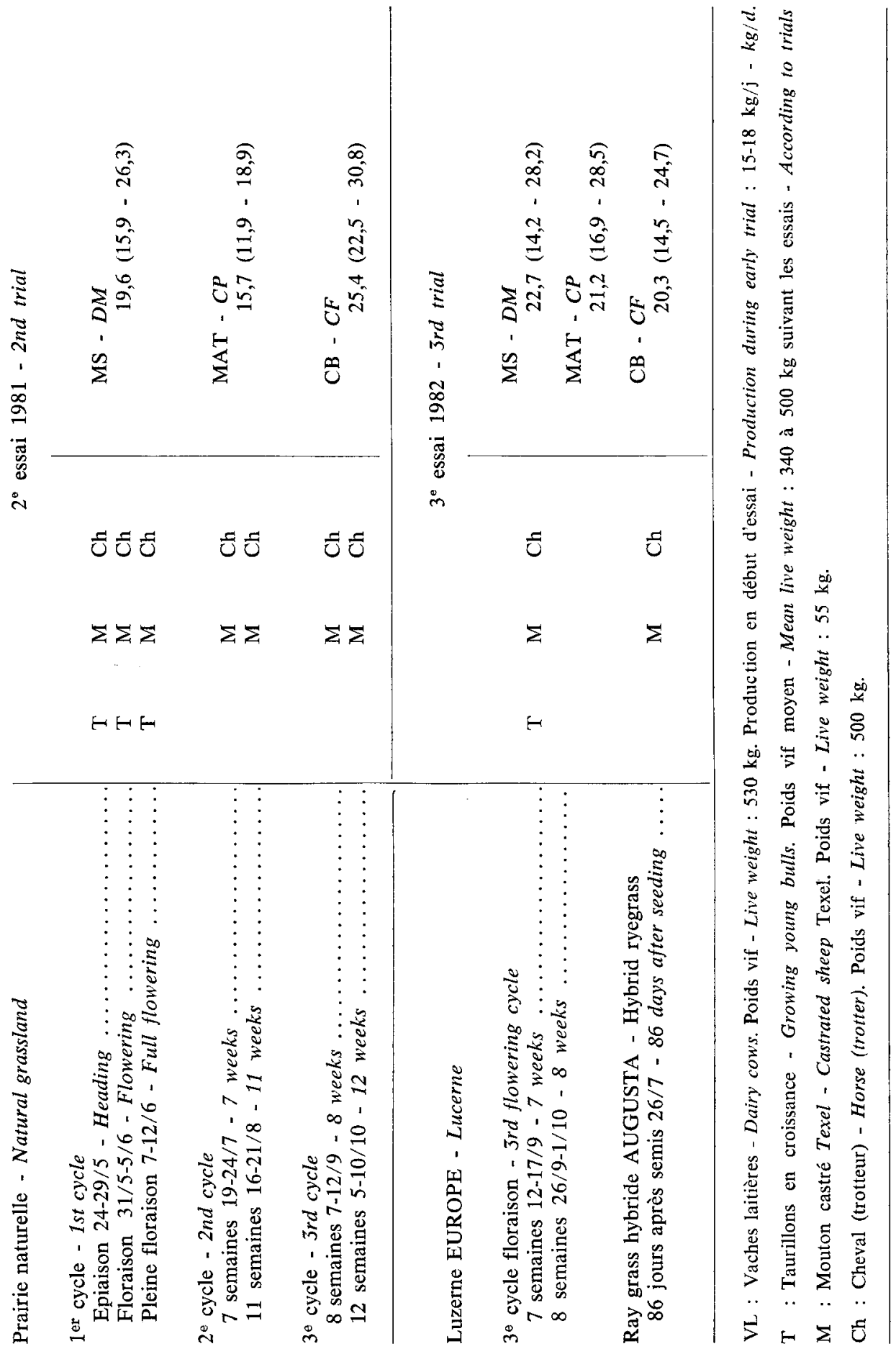


Les fèces étaient récoltées dans les bacs classiques équipant les stalles (taurillons, chevaux) et les cages (moutons). Les vaches étaient munies de sacs de collecte de fèces en buityle attachés à un dispositif étanche fixé à l'anus grâce à un harnais spécial. Les chevaux, maintenus en stalle longue, étaient équipés de tabliers en butyle permettant la récupération des crottins non piétinés.

\section{Mesures}

Les périodes de mesure de la digestibilité et des quantités ingérées ont duré 5 à 6 jours suivant les unités expérimentales auxquelles appartenaient les animaux ( 6 jours pour les moutons et les chevaux, 5 jours pour les taurillons et les vaches laitières).

Chaque période de mesure était précédée d'une période pré-expérimentale d'adaptation des animaux à leur régime. Dans le cas de mesures « en continu " (prairie naturelle), une semaine de mesure donnée servait de période d'adaptation au régime de la semaine suivante. Dans le cas de mesures isolées, les animaux étaient mis en régime pendant la semaine précédente sur une herbe dont les caractéristiques étaient aussi voisines que possible de l'herbe expérimentale.

On a déterminé la teneur en cendres, en matières azotées totales et en cellulose brute d'échantillons représentatifs de l'herbe offerte et refusée et des fèces correspondantes de la période de mesure. Nous avons également dosé l'NDF et l'ADF (VAN SoEST, 1967) des échantillons représentatifs du fourrage offert et des fèces correspondantes du $3^{\circ}$ essai.

\section{Résultats}

Le nombre de périodes de mesures communes aux trois espèces animales étant limité ( 6 sur 15 au total), nous avons analysé les résultats en considérant d'une part les comparaisons bovins/ovins $(\mathrm{n}=9)$ et d'autre part les comparaisons cheval/ovins $(n=12)$. Seules les droites de régression nous ont permis de les comparer simultanément.

\section{Digestibilité}

\section{Comparaison entre bovins et ovins}

Les résultats de digestibilité de la matière organique (dMO) sont regroupés dans le tableau 3. La dMO moutons a varié de 60,6 à 76,6 p. 100. La dMO bovins est significativement supérieure de 5,9 points à celle des moutons $(P<0,01)$. Cette différence varie de 1,8 à 7,7 points (luzernes) et ne semble pas dépendre de l'espèce botanique, du cycle ou de la composition chimique de l'herbe. La dMO bovins présente une liaison étroite ( $\mathrm{P}<0,01$ ) avec celle des ovins (fig. 1). On peut ainsi prévoir la dMO bovins à partir de celle des ovins avec une précision de 2,32 points. La différence de digestibilité entre bovins et ovins diminue quand la digestibilité du fourrage augmente, ceci malgré une luzerne dont la digestibilité sur bovins est faible et voisine de celle mesurée sur moutons. Les différences de dMO entre vaches laitières et moutons et entre taurillons et moutons sont du même ordre de grandeur. 


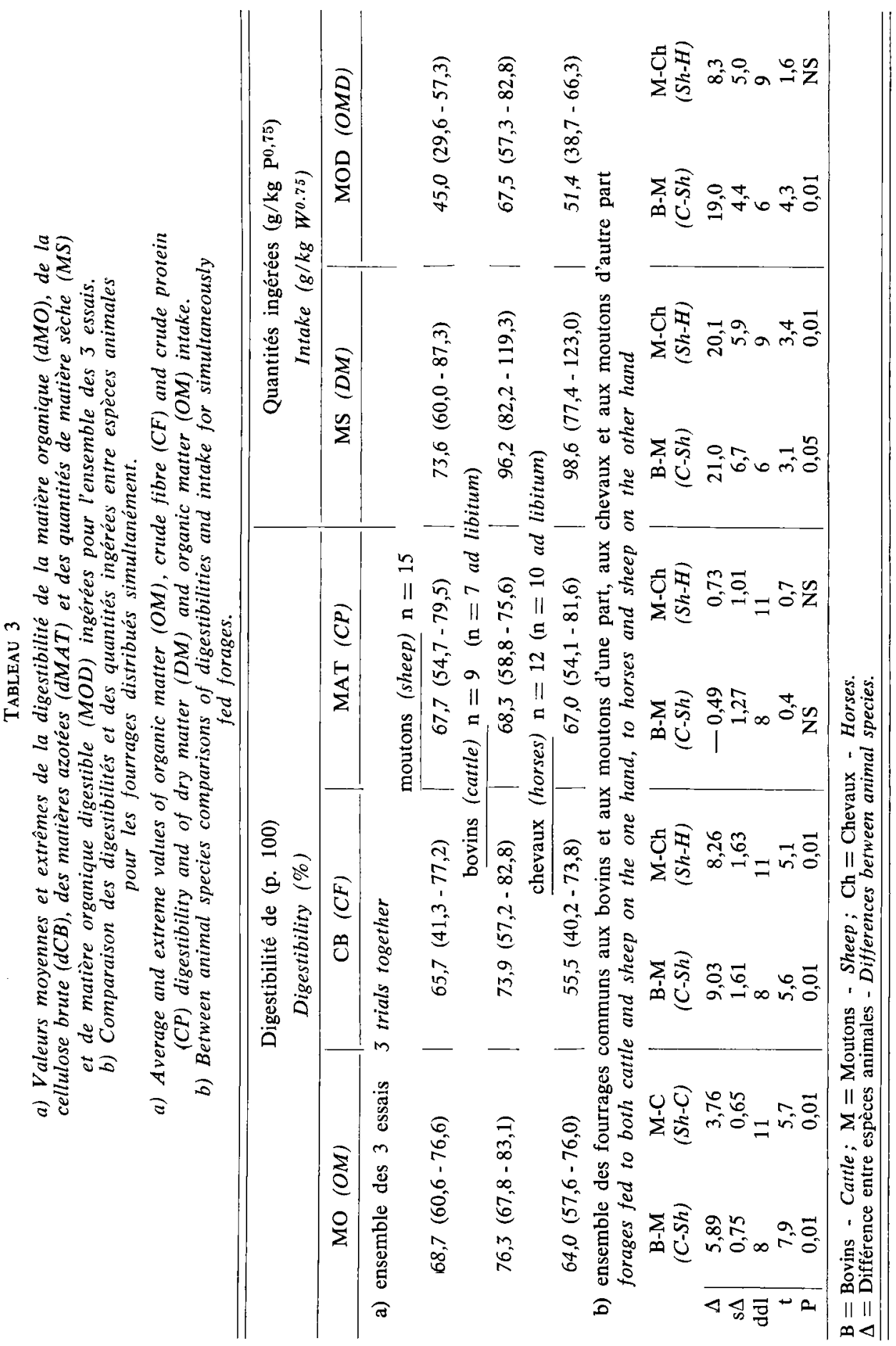




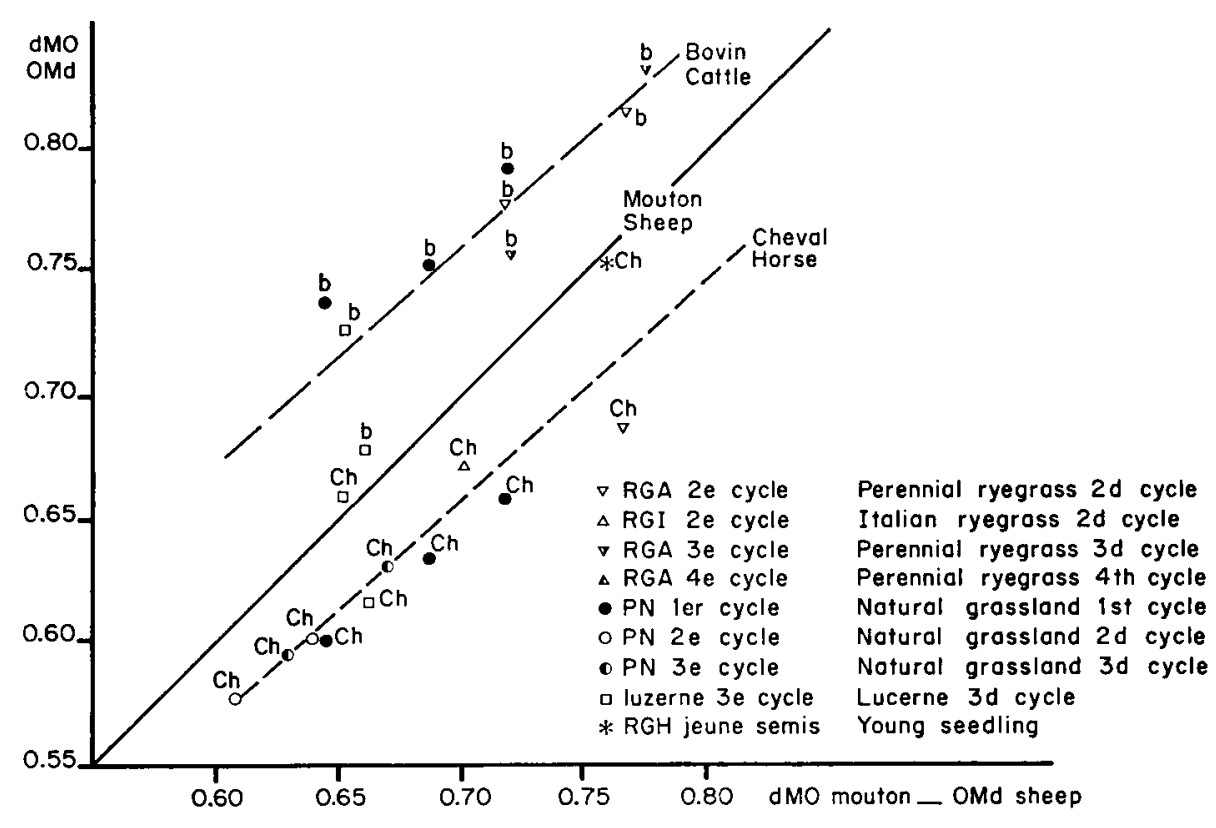

FIG. 1

Liaisons entre la digestibilité de la matière organique (dMO) bovin et cheval et la dMO ovins.

Relationship between cattle and horse organic matter digestibility (OMd) and sheep OMd.

$\mathrm{dMO}$ bovin $=14,39+0,880 \mathrm{dMO}$ mouton $\pm 2,32 ; \mathrm{n}=9 ; \mathrm{r}=0,866$.

OMd cattle $=14.39+0.880$ OMd sheep $\pm 2.32 ; n=9 ; r=0.866$.

$\mathrm{dMO}$ cheval $=3,39+0,890$ dMO mouton $\pm 2,31 ; \mathrm{n}=12 ; \mathrm{r}=0,901$.

oMd horse $=3.39+0.890$ OMd sheep $\pm 2.31 ; n=12 ; r=0.901$.

La digestibilité de la cellulose brute $(\mathrm{dCB})$ bovins est très significativement supérieure $(\mathrm{P}<0,01)$ à celle des moutons (tabl. 3). La différence (de 9,0 points en moyenne) varie de 3,3 ( $2^{\circ}$ cycle de ray-grass Anglais) à 16,4 points ( $3^{\circ}$ cycle de luzerne). La $\mathrm{dCB}$ bovins présente une liaison étroite avec la $\mathrm{dCB}$ ovins. Cette liaison est cependant moins étroite que dans le cas de la dMO (fig. 2). Les différences de dCB entre vaches laitières et moutons semblent moins élevées qu'entre taurillons et moutons mais le nombre de mesures est insuffisant pour pousser plus loin l'analyse. La digestibilité de la cellulose brute ou, d'une manière générale, la digestibilité des parois détermine la digestibilité de la matière organique; c'est ainsi que, comme pour la $\mathrm{dMO}$, la différence de $\mathrm{dCB}$ entre bovins et ovins a tendance à diminuer lorsque la $\mathrm{dCB} d u$ fourrage augmente (fig. 2).

La digestibilité des matières azotées (dMAT) bovins est voisine de la dMAT mouton (différence non significative, tabl. 3) ; la dMAT bovin présente une liaison significative avec la dMAT ovins (fig. 3). 


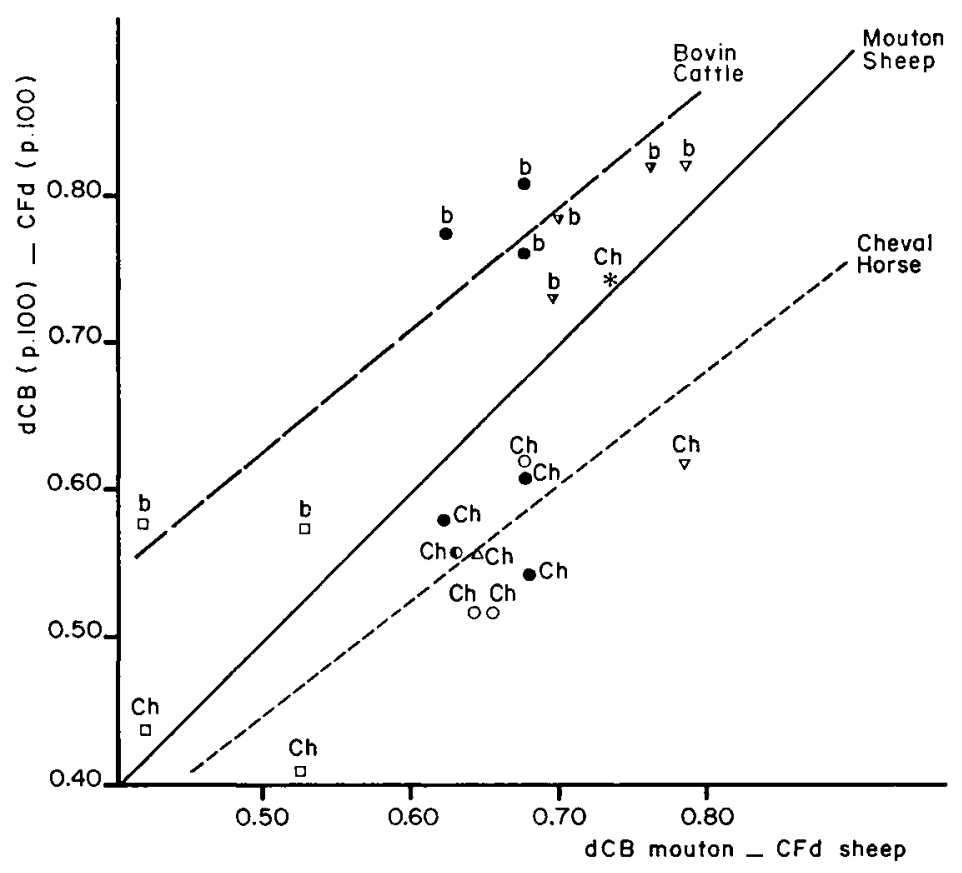

FIG. 2

Liaison entre la digestibilité de la cellulose brute (dCB) bovin et cheval et la dCB mouton. Relationship between cattle and horse crude fibre digestibility (CFd) and sheep $C F d$.

Mêmes symboles que la figure 1 .

Same signs as in figure 1.

$\mathrm{dCB}$ bovin $=23,36+0,78 \mathrm{dCB}$ mouton $\pm 4,40 ; \mathrm{n}=9 ; \mathrm{r}=0,908$.

Cattle $C F d=23.36+0.78$ sheep $C F d \pm 4.40 ; n=9 ; r=0.908$.

$\mathrm{dCB}$ cheval $=7,10+0,76 \mathrm{dCB}$ mouton $\pm 5,45 ; \mathrm{n}=12 ; \mathrm{r}=0,805$.

Horse $C F d=7.10+0.76$ sheep $C F d \pm 5.45 ; n=12 ; r=0.805$.

\section{Comparaison entre cheval et ruminants}

La dMO mouton est significativement supérieure $(\mathrm{P}<0,01)$ de 3,8 points à celle du cheval (tabl. 3). Cette différence varie de $-0,7$ (luzerne $3^{\mathrm{e}}$ cycle) à $+7,9$ points (ray-grass Anglais $2^{\circ}$ cycle, début épiaison). Il existe une relation étroite et significative entre dMO cheval et dMO mouton (fig. 1). Cette relation permet de prévoir la digestibilité cheval des fourrages verts à partir de la digestibilité mouton avec une bonne précision (2,31 points). A l'inverse de ce que l'on observe entre bovins et ovins, la différence de dMO entre mouton et cheval a tendance à diminuer pour les fourrages de faible digestibilité (fig. 1).

Nous avons porté sur la figure 4 l'évolution de la digestibilité de la prairie naturelle ( $2^{\circ}$ essai) au cours des différents cycles de croissance suivant les espèces animales. $\mathrm{Au}$ cours du $1^{\text {er }}$ cycle où les 3 espèces sont présentes on retrouve bien le classement taurillons, moutons, cheval par ordre de digestibilité décroissante. La dMO diminue plus rapidement avec le stade de croissance de la plante chez le mouton ( 7,2 points) que chez le cheval $(5,9$ points) et que chez le taurillon $(5,6$ points $)$. 


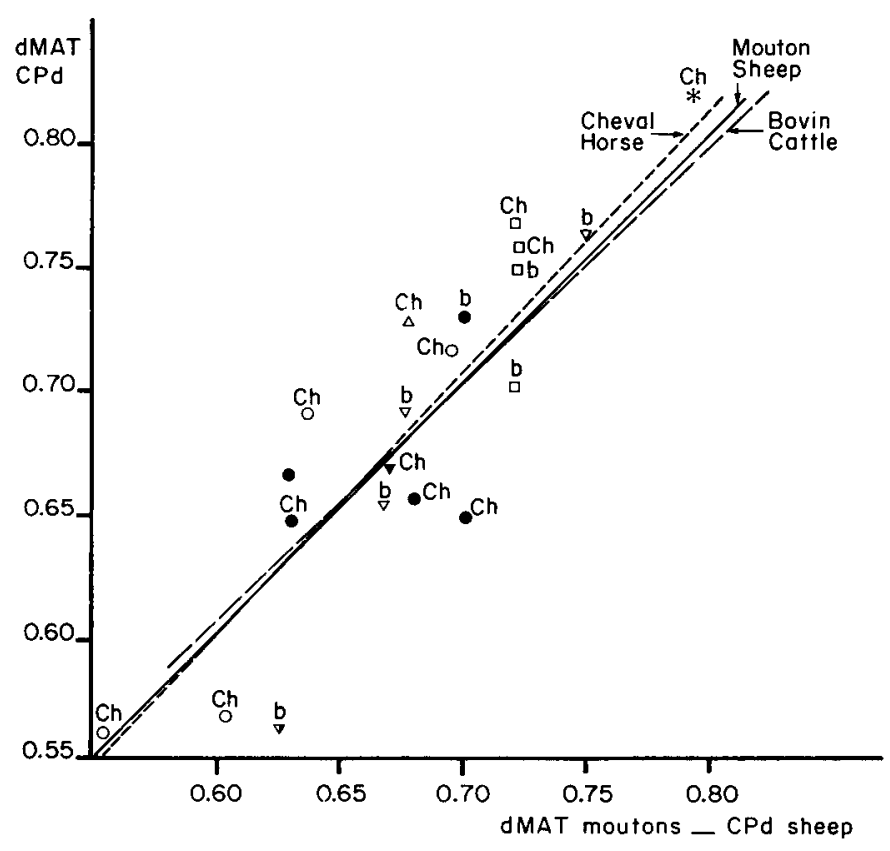

FIG. 3

Liaisons entre la digestibilité des matières azotées (dMAT) bovin et cheval et la dMAT mouton.

Relationship between cattle and horse crude protein digestibility $(C P d)$ and sheep CPd.

Mêmes symboles que la figure 1.

Same signs as in figure 1.

dMAT bovins $=5,69+0,91$ dMAT moutons $\pm 4,08 ; \mathrm{n}=9 ; \mathrm{r}=0,707$.

Cattle $C P d=5.69+0.91$ sheep $C P d \pm 4.08 ; n=9 ; r=0.707$.

dMAT cheval $=8,40+1,14$ dMAT moutons $\pm 3,56 ; \mathrm{n}=12 ; \mathrm{r}=0,905$.

Horse $C P d=8.40+1.14$ sheep $C P d \pm 3.56 ; n=12 ; r=0.905$.

La dCB mouton est significativement $(\mathrm{P}<0,01)$ supérieure de 8,26 points à la $\mathrm{dCB}$ cheval. La différence, voisine de celle observée entre bovins et ovins, a varié de $-2,4\left(3^{\circ}\right.$ cycle de luzerne) à 15,6 points ( $2^{\circ}$ cycle de ray-grass Anglais). La dCB cheval présente une liaison étroite avec la $\mathrm{dCB}$ mouton. Cette liaison est cependant moins étroite que dans le cas de la dMO (fig. 2). Comme chez les ruminants, c'est la digestibilité des parois qui détermine la digestibilité de la matière organique chez le cheval. Les digestibilités NDF et ADF (3 essai) conduisent, comme la dCB, au même classement : taurillons, moutons, chevaux par ordre de digestibilité décroissante (tab1. 4).

La dMAT cheval est voisine de la dMAT mouton (différence non significative, tab1. 3). Il existe une relation entre dMAT cheval et dMAT moutons; elle est plus étroite qu'entre bovins et ovins (fig. 3). Les différences de dMAT entre bovins et cheval sont très variables suivant le fourrage (elles varient de 8,0 points à $-5,7$ points ; leur moyenne est de 0,13 point). 


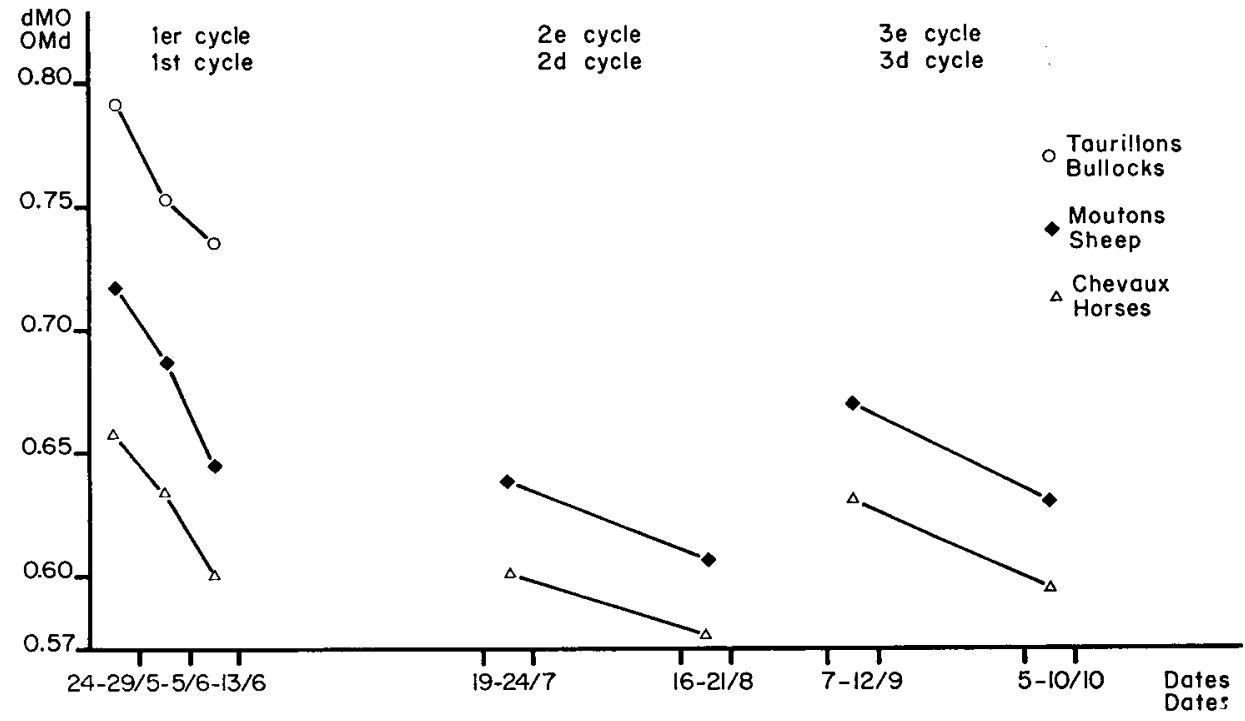

FiG. 4

Evolution, suivant le stade de végétation ( $1^{\text {er }}$ cycle) et l'âge des repousses $\left(2^{e}\right.$ et $3^{e}$ cycles), de la dMO chez les bovins, le cheval et le mouton (Prairie naturelle de Theix, 1981, essai 2).

Variation of OMd according to vegetative stage (1st cycle) and age of regrowths (2nd and 3rd cycles) in cattle, horse and sheep (natural grassland, Theix, 1981, trial 2).

\section{TABLEAU 4}

Comparaison de la digestibilité de NDF et de ADF entre mouton, cheval et taurillon. Comparison of NDF and ADF digestibility between sheep, horse and cattle (young bulls)

\begin{tabular}{|c|c|c|c|c|c|c|}
\hline & \multicolumn{2}{|c|}{$\begin{array}{c}\text { Luzerne } \\
3^{\mathrm{e}} \text { cycle }(7 \text { sem.) } \\
\text { Lucerne } \\
3 d \text { cycle (7 weeks) }\end{array}$} & \multicolumn{2}{|c|}{$\begin{array}{c}\text { Luzerne } \\
3^{\circ} \text { cycle }(8 \text { sem.) } \\
\text { Lucerne } \\
3 d \text { cycle (8 weeks) }\end{array}$} & \multicolumn{2}{|c|}{$\begin{array}{c}\text { Ray-grass hybride } \\
\text { (jeunes semis de } \\
13 \text { sem.) } \\
\text { Hybrid ryegrass } \\
\text { (13-week young } \\
\text { seeding) }\end{array}$} \\
\hline & NDF & $\mathrm{ADF}$ & NDF & ADF & NDF & ADF \\
\hline $\begin{array}{l}\text { Mouton } \\
\text { Sheep }\end{array}$ & 52,1 & 47,8 & 56,4 & 48,6 & 80,4 & 69,4 \\
\hline $\begin{array}{l}\text { Cheval } \ldots \ldots \ldots \ldots \\
\text { Horse }\end{array}$ & 52,2 & 42,7 & 50,2 & 37,5 & 77,3 & 67,5 \\
\hline $\begin{array}{l}\text { Taurillon .......... } \\
\text { Young bull }\end{array}$ & 66,8 & 62,5 & 59,3 & 56,2 & - & - \\
\hline
\end{tabular}

La variabilité de la dMO entre animaux est plus faible chez le mouton $(\mathrm{CV}=$ 1,8 p. 100) que chez le cheval $(\mathrm{CV}=2,6$ p. 100$)$, elle-même plus faible que chez les bovins $(\mathrm{CV}=4,4$ p. 100). Cette variabilité est indépendante de la nature de l'herbe. 
Liaisons, suivant l'espèce animale, entre la dMO et la composition chimique de l'herbe proposée (MAT, CB)

Il existe des relations entre dMO et MAT ( $r$ est compris entre 0,45 et 0,80 suivant l'espèce animale) ; elles sont plus étroites chez le cheval $(r=0,795)$ que chez les bovins $(r=0,539)$ et les ovins $(r=0,449)$, tableau 5 . Les liaisons entre $d M O$ et $C B$ sont plus étroites qu'entre dMO et MAT sauf chez le cheval, espèce pour laquelle la liaison $\mathrm{dMO} / \mathrm{CB}$ reste toutefois plus élevée que chez les bovins et le mouton. L'adjonction de MAT à $\mathrm{CB}$ n'améliore pas significativement les liaisons obtenues avec $\mathrm{CB}$ seule.

\section{Quantités ingérées}

Quantités de matière sèche ingérées

Les quantités de matière sèche ingérées (QI), exprimées dans la pratique en $\mathrm{kg} / 100 \mathrm{~kg}$ de poids vif ne sont pas significativement différentes d'une espèce à l'autre (tabl. 3) mais elles le sont quand on les exprime en $\mathrm{g} / \mathrm{kg} \mathrm{P0.75}$, expression recommandée par le $3^{\circ}$ Symposium du Métabolisme Energétique (Kleiber, 1965).

La variabilité $(\mathrm{Sx})$ des QI est en moyenne plus faible chez le mouton $( \pm 8,26)$ que chez les bovins $( \pm 14,72)$ et chez le cheval $( \pm 13,78)$.

Les QI bovins sont significativement supérieures (21 g) aux QI ovins (tabl. 3). Il n'existe pas de liaisons entre QI bovins et QI ovins. Il est vrai que nous ne disposons que de 7 mesures sur bovins, dont 4 sur vaches laitières et 3 sur taurillons.

Les QI cheval sont significativement $(\mathrm{P}<0,01)$ plus élevées que les $\mathrm{QI}$ moutons malgré la très grande variation des différences $\left(-9,9\right.$ à $\left.52,2 \mathrm{~g} / \mathrm{kg} \mathrm{P}^{0.75}\right)$; elles ne sont pas significativement différentes des QI bovins. Comme chez les bovins, elles diminuent beaucoup moins rapidement au cours du $1^{\text {er }}$ cycle d'exploitation de la prairie naturelle que chez les ovins.

Il existe des relations entre les quantités de matière sèche ingérées et la teneur en matières azotées ( $\mathrm{r}$ compris entre 0,406 et 0,621 ) ; elles sont moins étroites chez le cheval que chez les bovins et les ovins (tabl. 5). L'adjonction à MAT de CB n'améliore ces relations que dans le cas des bovins. Les quantités de matière sèche ingérées ne présentent aucune liaison avec la digestibilité sauf, toutefois, chez le mouton mais cette liaison n'est pas étroite $(r=0,478)$.

\section{Quantités de matière organique digestible ingérées}

La quantité de matière organique digestible ingérée (MOD) est la plus élevée chez les bovins et la plus faible chez les ovins (tabl. 3). Les différences entre bovins et ovins sont plus élevées $(19,0 \mathrm{~g})$ que celles entre cheval et mouton $(8,3 \mathrm{~g})$, cette dernière étant toutefois encore significative $(P<0,01)$.

\section{Discussion}

La digestibilité de la matière organique des fourrages est essentiellement déterminée par la digestibilité des constituants pariétaux. Celle-ci dépend de :

a) l'activité cellulolytique des microorganismes du rumen (ruminants) ou du cæcum-côlon (cheval) ; 
b) la durée de l'attaque des parois par ces microorganismes, cette dernière dépendant elle-même du temps de séjour des aliments dans le tube digestif.

\section{Comparaison bovins-ovins}

Nos résultats sur fourrages verts sont en accord avec ceux de la majorité des auteurs ayant expérimenté sur fourrages secs et avec ceux, plus rares, obtenus sur fourrages verts (tabl. 1). Ces résultats montrent que la $\mathrm{dCB}$ (et, par là, la $\mathrm{dMO}$ ) bovins est supérieure à la dCB moutons.

Nous n'avons pas pu effectuer de mesures de comportement alimentaire et de transit digestif. On sait cependant (CARLE \& DulPhy, 1980) que pour mastiquer une quantité donnée de fourrage les bovins mettent moins de temps que les ovins. On peut alors admettre que la réduction en fines particules est moins poussée chez les bovins. Ceci est d'ailleurs confirmé par le fait que la taille des particules de fourrage passant dans le feuillet est plus élevée chez les bovins que chez les ovins (GrENET, 1966 ; Thomas \& Campling, 1977 ; Michalet-Doreau, non publié). La digestibilité de la cellulose brute, plus élevée chez le bovin, résulterait donc vraisemblablement d'un temps de séjour plus long des aliments dans le tube digestif (notamment dans le réticulo-rumen) et d'une meilleure activité cellulolytique qui compenseraient ainsi l'intensité de mastication plus faible chez le bovin que chez le mouton.

\section{Tableau 5}

Précision des liaisons, suivant les espèces animales, entre la digestibilité de la matière organique $(d M O)$ de l'herbe ou les quantités de matière sèche qui en sont ingérées d'une part, et la teneur en matières azotées (MAT), seule ou associée à la teneur en cellulose brute $(C B)$ d'autre part.

Accuracy of relationship according to animals species between organic matter digestibility or dry matter intake of herbage and crude protein (CP) content of forage alone or associated with forage crude fibre content.

\begin{tabular}{|c|c|c|c|c|c|c|}
\hline \multirow{2}{*}{$\begin{array}{c}\text { Variables explicatives } \\
\text { (herbe offerte) } \\
\text { Explanative variable } \\
\text { (offered herbage) }\end{array}$} & \multicolumn{2}{|c|}{$\begin{array}{c}\text { Mouton - Sheep } \\
\mathrm{n}=13\end{array}$} & \multicolumn{2}{|c|}{$\begin{array}{c}\text { Bovin - Cattle } \\
\mathrm{n}=7\end{array}$} & \multicolumn{2}{|c|}{$\begin{array}{c}\text { Cheval - Horse } \\
\mathrm{n}=10\end{array}$} \\
\hline & $R(r)$ & Syx (Sx) & $R(r)$ & Syx (Sx) & $R(r)$ & Syx (Sx) \\
\hline & \multicolumn{6}{|c|}{$\begin{array}{c}\text { Digestibilité de la matière organique (p. 100) } \\
\text { Organic matter digestibility (\%) }\end{array}$} \\
\hline $\operatorname{MAT}($ p. $100 \mathrm{MO})-C P$ & 0,449 & $\pm 5,13$ & 0,539 & $\pm 3,16$ & 0,795 & $\pm 3,52$ \\
\hline $\mathrm{CB}$ (p. $100 \mathrm{MO})-C F \ldots$ & 0,490 & $\pm 5,0$ & 0,625 & $\pm 3,20$ & 0,705 & $\pm 4,11$ \\
\hline \multirow{2}{*}{$\begin{array}{l}\text { MAT, CB (p. } 100 \mathrm{MO}) \ldots \\
C P, C F\end{array}$} & 0,499 & $\pm 5,21$ & 0,639 & $\pm 3,23$ & 0,797 & $\pm 3,74$ \\
\hline & \multicolumn{6}{|c|}{$\begin{array}{c}\text { Quantité de matière sèche ingérée }\left(\mathrm{g} / \mathrm{kg} \mathrm{P}^{0,75}\right) \\
\text { Dry matter intake }\left(\mathrm{g} / \mathrm{kg} \mathrm{W}^{0.75}\right)\end{array}$} \\
\hline MS (p. 100) - DM & \multicolumn{2}{|c|}{ NS } & \multicolumn{2}{|c|}{ NS } & \multicolumn{2}{|c|}{ NS } \\
\hline MAT (p. $100 \mathrm{MO})-C P \ldots$ & 0,621 & $\pm 6,76$ & 0,612 & $\pm 12,75$ & 0,406 & $\pm 13,36$ \\
\hline $\begin{array}{l}\text { MAT, CB (p. } 100 \mathrm{MO}) \\
C P, C F\end{array}$ & 0,649 & $\pm 6,89$ & 0,918 & $\pm 7,15$ & 0,477 & $\pm 13,73$ \\
\hline dMO (p. 100) - OMd & 0,478 & $\pm 7,58$ & & $\mathbf{S}$ & & $\mathbf{S}$ \\
\hline
\end{tabular}


Comme dans le cas des foins (Carle \& Dulphy, non publié), la supériorité des bovins sur les ovins est d'autant plus élevée que la digestibilité des fourrages est faible.

Il est difficile d'interpréter les différences entre bovins et ovins dans les relations $\mathrm{dMO} / \mathrm{CB}, \mathrm{MAT}, \mathrm{CB}+\mathrm{MAT}$. Ces dernières sont moins étroites (tabl. 5) que celles généralement observées. Cela est vraisemblablement dû au nombre limité de nos mesures et, surtout, au fait que nous avons travaillé sur des fourrages d'espèces botaniques, de stade ou de cycle de croissance très différents les uns des autres.

Les quantités ingérées sont systématiquement plus élevées chez les bovins que chez les ovins. Ceci confirme les résultats de Demarouilly \& Weiss (1971). complétés par les données ayant servi à l'élaboration du système des unités d'encombrement (I.N.R.A., 1979). Il est également difficile d'interprétrer les différences entre espèces animales dans les relations quantités ingérées/composition chimique et digestibilité de l'herbe. En effet, outre la diversité «botanique » et le nombre réduit de mesures chez les bovins, nous avons utilisé des taurillons en croissance et des vaches laitières en milieu de lactation. Or on sait que le niveau de production et l'état physiologique de l'animal ont une influence très importante sur sa capacité d'ingestion.

\section{Comparaison cheval-ruminants}

Nos résultats de digestibilité sur fourrages verts sont en accord avec ceux, peu nombreux, obtenus sur foins par VAN DER Noot \& Gilbreath (1970) et Hintz (1969), tabl. 1.

Comme chez les ruminants, la digestibilité des fourrages chez le cheval est déterminée essentiellement par la digestibilité de leurs parois. Comme Martin-Rosset \& DorEau (non publié) et les quelques auteurs ayant effectué des mesures comparatives cheval/ruminants (tabl. 1), nous avons observé que la $\mathrm{dCB}$, et donc la dMO, sont moins élevées chez le cheval que chez les ruminants.

Toutefois, contrairement aux résultats de MARTin-Rosset \& DOREAu (non publié) et à ceux de VAN Der Noot \& Gilbreath (1970) obtenus sur fourrages conservés, les différences dans la dMO entre moutons et cheval diminuent lorsque la digestibilité de l'herbe diminue. Il convient de considérer cette observation avec prudence. En effet, la pente de l'équation de régression cheval/moutons a vraisemblablement été déviée en raison d'un résultat luzerne "sortant» de la moyenne et, surtout, du fait que la digestibilité de la majorité de nos échantillons était un peu élevée.

Pouvons-nous interpréter les différences de $\mathrm{dMO}$ et de $\mathrm{dCB}$ entre cheval et ruminants ?

Koller et al. (1978) utilisant les techniques de digestibilité in vitro et in sacco (fistule du rumen chez le bovin, fistule du cæcum chez le cheval), ont montré que la digestibilité de la matière organique et des constituants pariétaux de foin de luzerne, de dactyle et de fléole d'une part, et de paille de blé d'autre part, était plus importante lorsque les échantillons de ces fourrages étaient soumis (pendant 24 et 48 heures) à l'attaque des microorganismes du rumen que du cæcum. L'activité cellulolytique dans le cæcum est donc moins importante que dans le rumen.

On sait en outre (Axelsson, 1949 ; Haenlein, Holdren \& Yoon, 1966 ; Hintz, 1969) que la mastication totale (ingestion + rumination chez le ruminant) des ali- 
ments est moins poussée et que la vitesse de transit est plus élevée chez les chevaux que chez les ruminants. Le fait (Grenet, Martin-Rosset \& Chenost, 1985) que les particules végétales des fèces de cheval aient une taille supérieure et une concentration en lignine inférieure à celle des ruminants reflète d'ailleurs les différences entre espèces dans les phénomènes digestifs. La vitesse et l'intensité de la dégradation physique et fermentaire des parois sont donc moins élevées chez le cheval que chez les ruminants. Elles expliquent ainsi les différences de digestibilité observées entre cheval et ruminants.

Les quantités de matière sèche ingérées sont significativement plus élevées chez le cheval que chez le mouton; nous avions affaire dans les deux cas à des animaux voisins de l'entretien. En revanche, comme l'ont observé Martin-Rosset \& Doreau sur fourrages secs (non publié), elles sont voisines de celles ingérées par les bovins qui étaient, eux, soit en croissance soit en lactation. Nous n'avons pas pu mesurer la part qui revient à l'influence de l'état physiologique de l'animal sur sa capacité d'ingestion.

Il n'existe pratiquement pas de travaux ayant comparé l'ingestion chez le cheval et les ruminants alimentés à volonté. Les rares études conduites sur la régulation de l'ingestion chez le cheval (RALston, VAN Der BroecK \& BAILE, 1979) semblent indiquer que les aspects physiques sont moins importants chez le cheval que chez le ruminant. Cette régulation serait essentiellement d'ordre métabolique, comme chez les monogastriques non herbivores; les aspects physiques intervenant à un degré beaucoup moindre que chez les polygastriques. Ainsi, la vitesse de transit plus élevée, la vitesse de l'intensité de digestion plus faible chez le cheval que chez les ruminants, et par là, les phénomènes de vidange du tube digestif seraient des facteurs explicatifs des quantités ingérées moins importants que les aspects métaboliques. L'ingestion plus élevée chez le cheval peut aussi peut-être s'expliquer, tout au moins partiellement, par un besoin d'entretien plus élevé par rapport au métabolisme de base que celui des ruminants.

Enfin, toutes nos mesures ont été effectuées sur des animaux alimentés à volonté (à l'exception des deux luzernes) pour nous rapprocher des conditions d'alimentation au pâturage et pour comparer les QI entre espèces animales. Or, on sait que chez les ruminants, contrairement au cheval (MARTIN-RosSET \& DOREAU, non publié), le niveau d'alimentation a une influence sur la digestibilité (Blaxter, 1962 ; Leaver, Campling \& Holmes, 1969). Toutefois cette influence est plus importante dans le cas des fourrages conservés que dans celui des fourrages verts (ANDERSEN et al., 1959 ; RAYMOND, Harris \& Kemp, 1955). Il est donc peu probable que le niveau d'alimentation ad libitum ait pu, dans notre cas, modifier la digestibilité (tout au moins chez les ruminants) et par là, altérer les comparaisons entre espèces animales.

\section{Conclusion}

Ces premiers résultats sur fourrages verts sont encore fragmentaires. Ils complètent cependant ceux de Carle \& Dulphy (non publié) et de Martin-Rosset \& DoREAU (non publié) sur fourrages conservés. Ils permettent également de commencer à caractériser les relations $\mathrm{dMO}$ mouton/dMO bovin et cheval et, par là, les possibilités de transposition aux bovins et surtout au cheval (espèce sur laquelle nous manquons de données) de la dMO mesurée sur moutons. 
Il serait utile de poursuivre ces comparaisons selon des dispositifs expérimentaux appropriés :

- choix de quelques fourrages caractéristiques en ce qui concerne l'évolution de leur digestibilité et de leur ingestibilité ( 1 ou 2 graminées et 1 ou 2 légumineuses, prises aux stades où elles sont généralement exploitées dans la pratique);

- choix d'animaux consommateurs d'herbe utilisés à des états physiologiques et à des niveaux de production comparables d'une espèce à l'autre, de façon à pouvoir interpréter plus facilement les résultats et à mettre en évidence l'effet espèce et race sur les différences de digestibilité et de quantités ingérées observées.

\section{Summary}

\section{Comparison between species (sheep, cattle, horse) of digestibility and intake of green forages}

Contrary to preserved forages, only few comparisons are available between the digestibility and intake of green forages in sheep and cattle; comparisons between ruminants and horses are also scarce (Table 1). The purpose of this study was to compare the digestibility and level of intake of green forages in sheep, cattle and horses, green fed and on pasture. A total of 15 green forages was used (Table 2) offered alone green fed and ad libitum. Measurements lasted 5 days for cattle and horses and 6 days for sheep.

The ash, crude protein and crude fibre contents of grass (MATP, CBP) and faeces (MATF, CBF) samples, were determined.

\section{Digestibility}

Digestibility results of the organic matter (dMO), crude fibre $(\mathrm{dCB})$ and crude protein (dMAT) are given in Table 3. Cattle $\mathrm{dMO}$ and $\mathrm{dCB}$ were significantly higher than those of sheep. The digestibility differences between these two animal species were all the larger as the forage digestibility decreased (fig. 1 and 2). Digestibilities of NDF and ADF were lower in sheep than in cattle (Table 4). Cattle dMO could be predicted from sheep dMO with an accuracy of \pm 2.32 points $(r=0.866, n=9)$. Cattle and sheep dMAT were very similar (non significant difference) and they were closely related (fig. 3).

Horse dMO and $\mathrm{dCB}$ were lower than sheep dMO and dCB. The digestibility differences between these two species were all the smaller as the forage digestibility was low (Fig. 1 and 2). Digestibilities of NDF and ADF were lower in horse than in sheep (Table 4). Horse dMO could be predicted from sheep dMO with a correct accuracy $( \pm 2.31$ points, $\mathrm{n}=12, \mathrm{r}=0.901$ ). Horse and ruminant dMAT were very similar (non significant difference) and closely related (fig. 3).

Figure 4 shows the changes in natural pasture grass digestibility during its different growth cycles, according to animal species. Cattle, sheep and horse ranking by order of decreasing digestibility was observed again. The digestibility decrease during the 1st cycle was the most rapid in sheep and the less rapid in cattle, the horse being located in between. Decrease in dMO during regrowth was the same in horse and sheep. The dMO availability between animals of one and the same species was smallest in sheep and largest in cattle.

There were relationships between dMO and MAT in the three animal species but they were not strong (Table 5). They were closer in horse than in cattle and sheep. Relationships between $\mathrm{dMO}$ and CB were closer than between $\mathrm{dMO}$ and MAT except in the horse. In this species $\mathrm{dMO}$ was related to $\mathrm{CB}$, more closely than in sheep and cattle. Adding MAT to $\mathrm{CB}$ did not improve the $\mathrm{dMO}$ estimation accuracy.

Digestibility of dMO is determined in the three animal species by the digestibility of cell-wall constituents. Ranking of cattle, sheep and horse by decreasing order of digestibility could be explained by : 
1) The differences between species in the rate of passage and hence the intensity of the fermentative action in the plant cell-walls (cattle $<$ sheep $<$ horse).

2) The higher bacterial cellulolytic activity in the rumen than in the horse caecum.

\section{Feed intake}

The dry matter intake (QI, expressed in $\mathrm{g} / \mathrm{kg} \mathrm{W0.75)}$ in sheep was significantly $(P<0.01)$ lower than that of cattle or horses, the latter being not significantly different (Table 3). This agrees with the many results obtained in sheep and cattle with preserved forages, confirms the data recorded with green forages and completes the results (very few) of horse-ruminant comparisons.

The variability of $Q I$ between animals of the same species, the same in cattle and horse, was higher than in sheep. It was not possible to relate cattle or horse QI to sheep QI. The QI did not show any significant relationships with dMO except in the case of sheep.

The interpretation of these results was difficult as the number of measures available was restricted, the botanical variety and forage growth stage were very different the one from the others; in addition, the physiological stages of the animals were different and hence their feed ingestion ability. The differences between ruminants and horses could be explained by a different rate of passage but in particular by the fact that the regulation of feed intake in horses depends more on the metabolism than in ruminants. Further studies should be made with animals of comparable physiological stages and consuming simultancously a varied range of green forages.

This study allows to locate the horse and cattle relative to sheep generally used in digestibility measurements and to predict cattle and horse dMO from sheep dMO. It also shows the resemblance between the results obtained with preserved and green forages.

Key words : Comparison, interspecies, digestibility, green forages.

Reçu en juin 1984.

Accepté en juin 1985.

\section{Liste des abréviations}

$\begin{array}{ll}\text { MS } & \text { teneur en matière sèche } \\ D M & \text { dry matter content } \\ \text { MAT } & \text { teneur en matières azotées totales } \\ C P & \text { crude protein content } \\ \text { CB } & \text { teneur en cellulose brute } \\ C F & \text { crude fibre content } \\ N D F & \text { neutral detergent fibre (VAN SoEST, 1967) } \\ A D F & \text { acid detergent fibre (VAN SOEST, 1967) } \\ \text { dMO } & \text { digestibilité de la matière organique } \\ \text { OMd } & \text { organic matter digestibility } \\ \text { dMAT } & \text { digestibilité des matières azotées } \\ C P d & \text { crude protein digestibility } \\ \text { dCB } & \text { digestibilité de la cellulose brute } \\ C F d & \text { crude fibre digestibility } \\ \text { dNDF } & \text { digestibilité de l'NDF } \\ N D F d & \text { NDF digestibility } \\ \text { dADF } & \text { digestibilité de l'ADF } \\ A D F d & \text { ADF digestibility } \\ \text { QI } & \text { quantité de matière sèche ingérée } \\ D M I & \text { dry matter intake } \\ \text { MODI } & \text { quantité de matière organique digestible ingérée } \\ D O M I & \text { digestible organic matter intake } \\ \text { CV } & \text { coefficient de variation } \\ C V & \text { coefficient of variation }\end{array}$




\section{Références bibliographiques}

Alexander R.A., Hentges J.F., McCall J.T., Ash W.O., 1962. Comparative digestibility of nutrients in roughages by cattle and sheep. J. Anim. Sci., 21, 373-376.

Andersen P.E., Reid J.T., Anderson M.J., Stoud J.W., 1959. Influence of level of intake upon the apparent digestibility of forages and mixed diets by ruminants. J. Anim. Sci., 18, 1299-1307.

Axelsson J., 1949. The ability of cattle, sheep, horses and swine to digest the nutrients of the feeding stuffs. Kung. Lantbrukshogokolans. Ann., 16, 84-100.

BIRD P.R., 1974. Sulphur metabolism and excretion studies in ruminants. XIII - Intake and utilization of wheat straw by sheep and cattle. Austr. J. Agric. Res., 25, 631-642.

BLAXTER K.L., 1962. The energy metabolism of ruminants. Hutchinson, London, p. 329.

Blaxter K.L., Wainman F.W., 1964. The utilization of the energy of different rations by sheep and cattle for maintenance and for fattening. J. Agric. Sci., 63, 113-128.

Blaxter K.L., Wainman F.W., Davidson J.L., 1966. The voluntary intake of food by sheep and cattle in relation to their energy requirements for maintenance. Anim. Prod., 8, 75-83.

Buchman D.T., Hemken R.W., 1964. Ad libitum intake and digestibility of several alfalfa hay by cattle and sheep. J. Dairy Sci., 47, 861-864.

CAmeron C.D.T., 1966. The effect of nitrogen fertilizer application rates to grass on forage yields, body weight gains feed utilization and vitamine A status of steers. Can. J. Anim Sci., 46, 19-23.

Carle B., Dulphy J.P., 1980. Comportement alimentaire comparé des ovins et des bovins. Relation avec la digestibilité des aliments, Reprod. Nutr. Dévelop., 20 (5 B), 59-65.

Cipollini M.A., Schneider B.H., Lucas H.L., Pavlech H.M., 1951. Significance of the differences in digestibility of feeds by cattle and sheep. J. Anim. Sci., 10, 336-343.

Colovos N.F., Holter J.B., Koes R.M., Urban Jr. W.E., Davis H.A., 1970. Digestibility, nutritive value and intake of ensiled corn plant (Zea Mays) in cattle and sheep. I. Anim. Sci., 30, 819-824.

Demarquilly C., Weiss Ph., 1971. Liaisons entre les quantités de matière sèche de fourrages verts ingérées par les moutons et celles ingérées par les bovins. Ann. Zootech., 20, 119-134.

Donefer E., 1966. Collaborative in vivo studies on alfalfa hay. J. Anim. Sci., 25, 12271231.

Engels E.N.A., Ferreira B., Swart J.A., Nieman P.J., 1978. Comparative feed intake and digestibility studies with sheep and cattle on roughages. South. Afric. J. Anim. Sci., 8, 149-152.

Greenhalgh J.F.D., Reid G.W., 1973. The effect of pelleting various diets on intake and digestibility in sheep and cattle. Anim. Prod., 16, 223-233.

Grenet E., 1966. Les particules végétales des fèces de moutons. Ann. Zootech., 16, 303312 .

Grenet E., Martin-Rosset W., Chenost M., 1985. Compared size and structure of plant particules in the horse and the sheep faeces (à paraître).

Haenlein C.F.W., Holdren R.D., Yoon Y.M., 1966. Comparative response of horses and sheep to different physical forms of alfalfa hays. J. Anim. Sci., 25, 740-743.

Hintz H.F., 1969. Review article : Equine nutrition. Comparisons of digestion coefficients obtained with cattle, sheep, rabbits and horses. The veterinarian, 6, 45-51.

I.N.R.A., Département Elevage des Ruminants, 1979. Le système des unités d'encombrement pour les bovins. Bull. Techn. C.R.Z.V. Theix, I.N.R.A., 38, 57-79.

JoHnsson S., 1972. Digestibility experiments with growing bulls and wethers consuming artificially dried grass. Swedish J. Agric. Res., 2, 97-103. 
KLEIBER M., 1965. Metabolic body size. In 3rd Symposium of Energy Metabolism, 427-435. Ed. KL Blaxter, London. New York, Academic Press.

Koller B.L., Hintz H.F., Robertson J.B., Van Soest, 1978. Comparative cell wall and dry matter digestion in the caecum of the poney and the rumen of the cow using in vitro and nylon bag techniques. J. Anim. Sci., 47 (1), 209-215.

Leaver J.D., Campling R.C., Holmes W., 1969. The effect of level of feeding on the digestibility of diet for sheep and cattle. Anim. Prod., 11, 11-18.

PlaYNe M.J., 1978. Differences between cattle and sheep in their digestion and relative intake of a mature tropical grass hay. Anim. Feed Sci. Technol, 3 (1), 41-49.

Raymond W.F.C., Harris C.E., Kemp C.D., 1955. Studies in the digestibility of herbage. VI - The effect of level of herbage intake on the digestibility of herbage by sheep. $J$. Br. Grassl. Soc., 10, 19-26

Ralston S.L., Van Der Broeck G., Baile C.A., 1979. Feed intake patterns and associated blood glucose free fatty acids and insulin changes in ponies. J. Anim. Sci., 49, 838-845.

Rees M.C., Little D.A., 1980. Differences between sheep and cattle in digestibility, voluntary intake and retention time in the rumen of three tropical grasses. J. Agric. Sci., 94 (2), 483-485.

Samsher Jang, Majmudar B.N., 1962. A study on comparative digestibilities in different species of ruminants. Ann. Biochem. Exp. Med., 22 (12), 303-308.

SChNeIDER B.H., FlatT W.A., 1965. In The evaluation of feeds through digestibility experiments. Athens, Georgia : University of Georgia Press.

Siebert B.D., KenNedy P.M., 1972. The utilization of spear grass (Heteropogon contortus). I - Factors limiting intake and utilization by cattle and sheep. Austr. J. Agric. Res., 23, 35-44.

Thomas S., Campling R.C., 1977. Comparison of some factors affecting digestibility in sheep and cows. J. Br. Grassld Soc., 32, 33-41.

Van Der Noot G.W., Gilbreath E.B., 1970. Comparative digestibility of components of forages by geldings and steers. J. Anim. Sci., 31, 351-355.

VAN Der Noot G.W., Cordts R.H., Hunt R., 1965. Comparative nutrient digestibility of silages by cattle and sheep. J. Anim. Sci., 24, 47-50.

VAN SoEsT P.J., 1967. Development of a comprehensive system of feed analyses and its application to forages. J. Anim., Sci., 28, 119-128. 